\title{
Numerical analysis of the advection-diffusion problems in thin curvilinear channel based on multiscale finite element method
}

\author{
Mazuriak N., Savula Ya. \\ Ivan Franko National University of Lviv \\ 1 Universytetska str., 79000, Lviv, Ukraine
}

(Received 17 June 2017)

\begin{abstract}
The advection-diffusion problem in a thin curvilinear channel is considered. The multiscale finite element method is applied to solving the formulated model problem. It is shown that this method is efficient in the case of sufficiently large Peclet numbers. Numerical examples are presented and analysed.
\end{abstract}

Keywords: multiscale finite element method, advection-diffusion, thin curvilinear channel.

2000 MSC: $65 \mathrm{M} 55,65 \mathrm{M} 60$

UDC: $517.958: 519.6$

\section{Introduction}

The process of mathematical modeling of modern scientific and technical problems such as heat transfer problems, transport of pollutants and other problems often requires investigation of transfer of substance in the environment with complex heterogeneous structure. A lot of investigating domains are characterized by the availability of thin channels with properties that are different from the main environment. These heterogeneities produce significant difficulties while using the known approaches to solving given problems. Besides this, most of such problems involves differences in types of transfer of substance in thin channels. The simplest of these is the existence of advective transfer together with the diffusive one. It is known that solving the advection-diffusion problem by numerical methods, in particular by the finite element method (FEM) [1], becomes highly complicated in the case of large Peclet numbers because of the instability of computational process.

The multiscale finite element method (MsFEM) [2] was introduced as a modification of FEM for diffusion problems in the heterogeneous environment. The main idea of MsFEM is that the multiscale structure of the solution is incorporated into the specific localized basis functions. Thereby the multiscale features of the solution are captured, and thus better approximation with a small number of finite elements takes place. It was shown [3,4] that MsFEM is also efficient for solving advectiondiffusion problems in the heterogeneous environment. In this paper MsFEM is applied to solving the advection-diffusion problems with large Peclet numbers in a thin curvilinear channel, that is in the homogeneous environment.

\section{Multiscale finite element method}

Multiscale finite element method consists of two steps: construction of the multiscale basis functions and coupling these multiscale basis functions into global numerical formulation. The basis functions are designed to capture the specific features of the solution (important features of the solution are incorporated into these localized basis functions). A global formulation couples these basis functions to provide an accurate approximation of the solution. 
Let us consider the following one-dimensional problem

$$
\begin{aligned}
& L u=f \quad \text { in } \quad \Omega, \\
& u=0 \quad \text { on } \quad \partial \Omega,
\end{aligned}
$$

where $L$ is the differential operator of the problem (we will consider the advection-diffusion operator), $u$ is the unknown function, $f$ is the given function, $\Omega=[a, b] \subset \mathbb{R}$.

At first, we consider the basis functions construction. Let $P_{h}$ be a partition of the interval $[a, b]$ into finite elements by points $x_{i}, i=\overline{0, n}, x_{0}=a, x_{n}=b$. We call this partition the coarse grid and assume that the coarse grid can be resolved via a finer resolution called the fine grid. Let $\varphi_{i}^{0}$ be the nodal basis functions of FEM. For simplicity, one can assume that $\varphi_{i}^{0}$ are piecewise linear functions [1]. Let us denote as $S_{i}$ the support of $\varphi_{i}^{0}$ and define MsFEM basis functions $\varphi_{i}$ with support in $S_{i}$ as follows

$$
L \varphi_{i}=0 \quad \text { in } K, \quad \varphi_{i}=\varphi_{i}^{0} \quad \text { on } \quad \partial K \quad \forall K \in P_{h}, \quad K \subset S_{i}, \quad i=\overline{0, n}
$$

that is multiscale basis functions coincide with standard finite element basis functions on the boundaries of a coarse-grid block $K$ and capture the specific features of the solution in the interior of each coarsegrid block. Throughout, $K$ denotes a coarse-grid block.

Next, we consider the global formulation of MsFEM. Analogously to standard FEM we will look for the solution in the form

$$
\tilde{u}(x)=\sum_{i=1}^{n} u_{i} \varphi_{i}(x) .
$$

We introduce the space

$$
\tilde{V}=\left\{u(x): u(x) \in W_{2}^{(1)}, u(a)=0, u(b)=0\right\} .
$$

After multiplying the equation (1) on the arbitrary function $v \in \tilde{V}$ and integrating the result on $\Omega$ we will obtain the variational formulation of the problem (1)-(2) that is as follows. Find such function $u(x) \in \tilde{V}$ that satisfies the condition

$$
(L u, v)=(f, v) \quad \forall v \in \tilde{V} .
$$

In the case of advection-diffusion operator $L$ we can get rid of second derivatives in (5) using integration of $(L u, v)$ by parts.

We take in (5) $v=\varphi_{j}^{0} \in \tilde{V}, j=\overline{1, n}$. Substituting (4) into (5) we obtain the following system of equations

$$
A \bar{u}=b,
$$

where $A=\left(a_{i j}\right)$ is the matrix with elements $a_{i j}=\sum_{r=1}^{n} \int_{K_{r}} L \varphi_{i}(x) \varphi_{j}^{0}(x) d x, i, j=\overline{1, n} ; \overline{\boldsymbol{u}}=\left(u_{i}\right)$ is the vector of unknown values of the function $u$ in nodes $x_{i} \in P_{h}, i=\overline{1, n} ; b=\left(b_{j}\right)$ is the vector with elements $b_{j}=\int_{a}^{b} f(x) \varphi_{j}^{0}(x) d x, j=\overline{1, n}$.

As a conclusion let us present the algorithm of MsFEM [2]

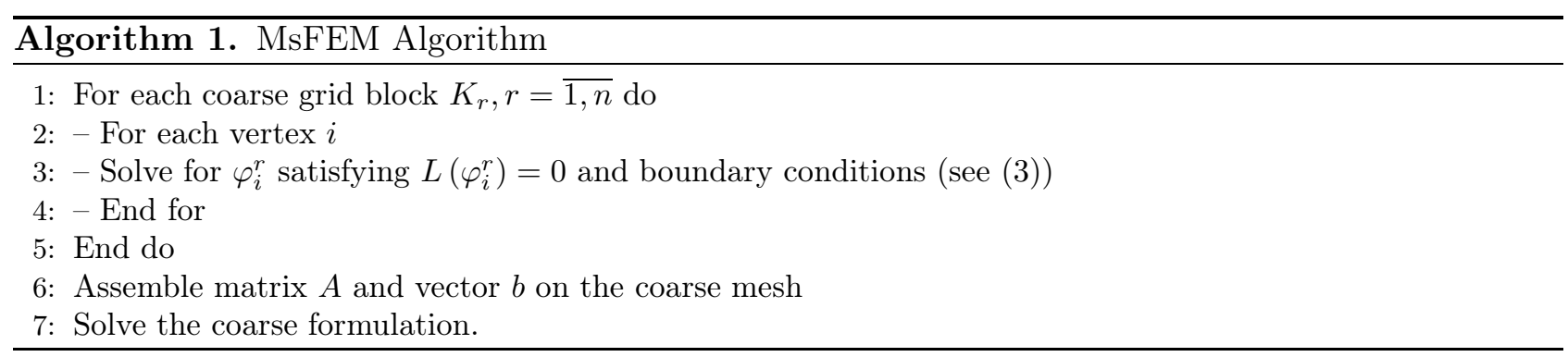




\section{Mathematical model of advection-diffusion in a thin curvilinear channel}

Let some smooth curve is defined by the parametric equation

$$
x_{1}=x_{1}\left(\alpha_{1}\right), \quad x_{2}=x_{2}\left(\alpha_{1}\right) .
$$

We construct the curvilinear coordinate system $\alpha_{1}, \alpha_{2}$, that is related to (6) so coordinate $\alpha_{1}$ corresponds to the direction of curve tangent and coordinate $\alpha_{2}$ corresponds to the direction of curve normal.

Let us consider a thin curvilinear channel (see Fig. 1) in the coordinate system introduced above

$$
\Omega=\left\{\alpha_{1} \alpha_{2}: \alpha_{1}^{b} \leqslant \alpha_{1} \leqslant \alpha_{1}^{e},-h \leqslant \alpha_{2} \leqslant h\right\}
$$

We denote by $A$ the Lame coefficient of the curve (6) and by $K-$ the curvature of this curve [5]

$$
A=\sqrt{\left(x_{1}^{\prime 2}+x_{2}^{\prime 2}\right)}, \quad K=\frac{x_{2}^{\prime \prime} x_{1}^{\prime}-x_{1}^{\prime \prime} x_{2}^{\prime}}{A^{3}} .
$$

The components $g_{i j}$ of the metric tensor in orthogonal coordinate system $\alpha_{1} \alpha_{2}$ can be represented as

$$
g_{11}=H^{2}, \quad g_{22}=1, \quad g_{12}=g_{21}=0 ; \quad H=A\left(1+\alpha_{2} K\right) .
$$

Let us consider the advection-diffusion equation in $\Omega$

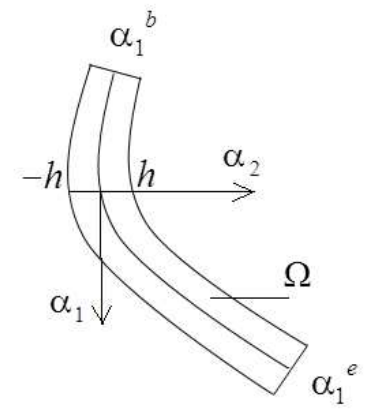

Fig. 1. Thin curvilinear channel.

$$
\kappa \frac{\partial u}{\partial \tau}+\kappa \boldsymbol{W} \operatorname{grad} u-\operatorname{div}(\lambda \operatorname{grad} u)=f,
$$

where $u\left(\tau, \alpha_{1}, \alpha_{2}\right)$ is the unknown function, $\boldsymbol{W}=\{P e, 0\}, P e$ is the Peclet number, $\kappa=$ const $\geqslant 0$, $\lambda=$ const $\geqslant 0$ are the given coefficients, $\tau$ is the time, $f\left(\alpha_{1}, \alpha_{2}\right)$ is the given function.

We take into account that the following equations will take place in orthogonal coordinate system $\alpha_{1} \alpha_{2}$

$$
\begin{gathered}
\operatorname{div}(\lambda \operatorname{grad} u)=\frac{1}{\sqrt{g}}\left(\frac{\partial}{\partial \alpha_{1}}\left(\frac{\sqrt{g}}{g_{11}} \lambda \frac{\partial u}{\partial \alpha_{1}}\right)+\frac{\partial}{\partial \alpha_{2}}\left(\frac{\sqrt{g}}{g_{22}} \lambda \frac{\partial u}{\partial \alpha_{2}}\right)\right), \\
\operatorname{grad} u=\left\{\frac{1}{\sqrt{g_{11}}} \frac{\partial u}{\partial \alpha_{1}}, \frac{1}{\sqrt{g_{22}}} \frac{\partial u}{\partial \alpha_{2}}\right\}, \\
g=\operatorname{det}\left\{g_{i j}\right\} .
\end{gathered}
$$

According to (7) we obtain

$$
\begin{gathered}
\operatorname{div}(\lambda \operatorname{grad} u)=\frac{1}{H} \frac{\partial}{\partial \alpha_{1}}\left(\frac{\lambda}{H} \frac{\partial u}{\partial \alpha_{1}}\right)+\frac{1}{H} \frac{\partial}{\partial \alpha_{2}}\left(\lambda H \frac{\partial u}{\partial \alpha_{2}}\right), \\
\operatorname{grad} u=\left\{\frac{1}{H} \frac{\partial u}{\partial \alpha_{1}}, \frac{\partial u}{\partial \alpha_{2}}\right\}, \\
\sqrt{g}=H .
\end{gathered}
$$

Thereby we can write equation (8) in the form

$$
\kappa \frac{\partial u}{\partial \tau}+\kappa \frac{P e}{H} \frac{\partial u}{\partial \alpha_{1}}-\frac{1}{H} \frac{\partial}{\partial \alpha_{1}}\left(\frac{\lambda}{H} \frac{\partial u}{\partial \alpha_{1}}\right)-\frac{1}{H} \frac{\partial}{\partial \alpha_{2}}\left(\lambda H \frac{\partial u}{\partial \alpha_{2}}\right)=f .
$$


We consider the following boundary and initial conditions

$$
\begin{gathered}
\left.u\right|_{\alpha_{1}=\alpha_{1}^{b}}=0, \\
\left.u\right|_{\alpha_{1}=\alpha_{1}^{e}}=0, \\
-\left.\lambda \frac{\partial u}{\partial \alpha_{2}}\right|_{\alpha_{2}=h}=q^{+}, \\
-\left.\lambda \frac{\partial u}{\partial \alpha_{2}}\right|_{\alpha_{2}=-h}=q^{-}, \\
u\left(0, \alpha_{1}, \alpha_{2}\right)=u^{(0)}\left(\alpha_{1}, \alpha_{2}\right),
\end{gathered}
$$

where $q^{+}\left(\tau, \alpha_{1}, h\right), q^{-}\left(\tau, \alpha_{1},-h\right), u^{(0)}\left(\alpha_{1}, \alpha_{2}\right)$ are the given functions.

We assume that the channel is thin, that is $h K \ll 1$. Under this assumption we will look for the solution in the form of linear distribution over the variable $\alpha_{2}$

$$
u=u_{1}\left(\tau, \alpha_{1}\right)+\frac{\alpha_{2}}{h} u_{2}\left(\tau, \alpha_{1}\right)
$$

where $u_{1}\left(\tau, \alpha_{1}\right), u_{2}\left(\tau, \alpha_{1}\right)$ are the unknown functions.

We consider the following space

$$
V=\left\{\psi\left(\alpha_{1}\right) \mid \psi\left(\alpha_{1}\right) \in W_{2}^{(1)}\left(\alpha_{1}^{b}, \alpha_{1}^{e}\right), \psi\left(\alpha_{1}^{b}\right)=0, \psi\left(\alpha_{1}^{e}\right)=0\right\} .
$$

Let us substitute (13) into (9) and orthogonalize residuals to arbitrary functions $\psi\left(\alpha_{1}\right) \in V$ and $\alpha_{2} \psi\left(\alpha_{1}\right) \in V$ in terms of integrals over the variables $\alpha_{1}, \alpha_{2}$ in limits from $\alpha_{1}^{b}$ to $\alpha_{1}^{e}$ and from $-h$ to $h$ respectively. We will obtain the following equations

$$
\begin{gathered}
m\left(u^{\prime}, \psi\right)+a(u, \psi)+b(u, \psi)=l(\psi), \\
m\left(u^{\prime}, \alpha_{2} \psi\right)+a\left(u, \alpha_{2} \psi\right)+b\left(u, \alpha_{2} \psi\right)=l\left(\alpha_{2} \psi\right),
\end{gathered}
$$

where

$$
\begin{aligned}
m\left(u^{\prime}, \psi\right)= & \int_{\alpha_{1}^{b}}^{\alpha_{1}^{e}} \int_{-h}^{h} \kappa\left(\frac{\partial u_{1}}{\partial \tau}+\frac{\alpha_{2}}{h} \frac{\partial u_{2}}{\partial \tau}\right) \psi A\left(1+\alpha_{2} K\right) d \alpha_{1} d \alpha_{2}, \\
a(u, \psi)= & \int_{\alpha_{1}^{b}}^{\alpha_{1}^{e}} \int_{-h}^{h} \kappa P e \frac{1}{H}\left(\frac{\partial u_{1}}{\partial \alpha_{1}}+\frac{\alpha_{2}}{h} \frac{\partial u_{2}}{\partial \alpha_{1}}\right) \psi H d \alpha_{1} d \alpha_{2}, \\
b(u, \psi)= & -\int_{\alpha_{1}^{b}}^{\alpha_{1}^{e}} \int_{-h}^{h}\left(\frac{\partial}{\partial \alpha_{1}}\left(\frac{\lambda}{A}\left(\frac{\partial u_{1}}{\partial \alpha_{1}}-\alpha_{2} K \frac{\partial u_{1}}{\partial \alpha_{1}}+\frac{\alpha_{2}}{h} \frac{\partial u_{2}}{\partial \alpha_{1}}-\frac{\alpha_{2}^{2} K}{h} \frac{\partial u_{2}}{\partial \alpha_{1}}\right)\right)\right) \psi d \alpha_{1} d \alpha_{2} \\
& -\int_{\alpha_{1}^{b}}^{\alpha_{1}^{e}} \int_{-h}^{h}\left(\frac{\partial}{\partial \alpha_{2}}\left(\lambda A\left(1+\alpha_{2} K\right) \frac{\partial u}{\partial \alpha_{2}}\right)\right) \psi d \alpha_{1} d \alpha_{2}, \\
l(u, \psi)= & \int_{\alpha_{1}^{b}}^{\alpha_{1}^{e}} \int_{-h}^{h} f \psi A\left(1+\alpha_{2} K\right) d \alpha_{1} d \alpha_{2} .
\end{aligned}
$$

Let us integrate these components over the variable $\alpha_{2}$. Taking into account our assumption of $h K \ll 1$ we will neglect components that contain $\left(\alpha_{2} K\right)^{2}$. After using (11) we obtain

$$
m\left(u^{\prime}, \psi\right)=\int_{\alpha_{1}^{b}}^{\alpha_{1}^{e}} 2 h \kappa\left(\frac{\partial u_{1}}{\partial \tau}+\frac{K h}{3} \frac{\partial u_{2}}{\partial \tau}\right) \psi A d \alpha_{1},
$$




$$
\begin{aligned}
m\left(u^{\prime}, \alpha_{2} \psi\right)= & \int_{\alpha_{1}^{b}}^{\alpha_{1}^{e}} \frac{2 h^{2}}{3} \kappa\left(\frac{\partial u_{2}}{\partial \tau}+K h \frac{\partial u_{1}}{\partial \tau}\right) \psi A d \alpha_{1} \\
a(u, \psi)= & \int_{\alpha_{1}^{b}}^{\alpha_{1}^{e}} 2 h \kappa P e \frac{\partial u_{1}}{\partial \alpha_{1}} \psi d \alpha_{1} \\
a\left(u, \alpha_{2} \psi\right)= & \int_{\alpha_{1}^{b}}^{\alpha_{1}^{e}} \frac{2 h^{2}}{3} \kappa P e \frac{\partial u_{2}}{\partial \alpha_{1}} \psi d \alpha_{1} \\
b(u, \psi)= & -\int_{\alpha_{1}^{b}}^{\alpha_{1}^{e}}\left(\frac{\partial}{\partial \alpha_{1}}\left(\frac{2 h \lambda}{A}\left(\frac{\partial u_{1}}{\partial \alpha_{1}}-\frac{K h}{3} \frac{\partial u_{2}}{\partial \alpha_{1}}\right)\right)\right) \psi d \alpha_{1} \\
& -\int_{\alpha_{1}^{b}}^{\alpha_{1}^{e}}\left(-A(1+K h) q^{+}+A(1-K h) q^{-}\right) \psi d \alpha_{1} \\
b\left(u, \alpha_{2} \psi\right)= & -\int_{\alpha_{1}^{b}}^{\alpha_{1}^{e}}\left(\frac{\partial}{\partial \alpha_{1}}\left(\frac{2 h^{2}}{3} \frac{\lambda}{A}\left(\frac{\partial u_{2}}{\partial \alpha_{1}}-K h \frac{\partial u_{1}}{\partial \alpha_{1}}\right)\right)-2 \lambda A u_{2}\right) \psi d \alpha_{1} \\
& -\int_{\alpha_{1}^{b}}^{\alpha_{1}^{e}}\left(-A(1+K h) h q^{+}-A(1-K h) h q^{-}\right) \psi d \alpha_{1} .
\end{aligned}
$$

As $\psi\left(\alpha_{1}\right)$ is arbitrary, we can write the basic equations for finding $u_{1}$ and $u_{2}$ [6]

$$
\begin{aligned}
\kappa\left(\frac{\partial u_{1}}{\partial \tau}+\frac{K h}{3} \frac{\partial u_{2}}{\partial \tau}\right)+\frac{\kappa P e}{A} \frac{\partial u_{1}}{\partial \alpha_{1}}- & \frac{1}{A} \frac{\partial}{\partial \alpha_{1}} \frac{\lambda}{A}\left(\frac{\partial u_{1}}{\partial \alpha_{1}}-\frac{K h}{3} \frac{\partial u_{2}}{\partial \alpha_{1}}\right) \\
& =\frac{1}{2 h}\left(\int_{-h}^{h} f\left(1+\alpha_{2} K\right) d \alpha_{2}-(1+K h) q^{+}+(1-K h) q^{-}\right),
\end{aligned}
$$

$$
\begin{aligned}
\frac{h}{3} \kappa\left(\frac{\partial u_{2}}{\partial \tau}+K h \frac{\partial u_{1}}{\partial \tau}\right)+\frac{h}{3} \frac{\kappa P e}{A} & \frac{\partial u_{2}}{\partial \alpha_{1}}-\frac{h}{3} \frac{1}{A} \frac{\partial}{\partial \alpha_{1}} \frac{\lambda}{A}\left(\frac{\partial u_{2}}{\partial \alpha_{1}}-K h \frac{\partial u_{1}}{\partial \alpha_{1}}\right)+\frac{\lambda}{h} u_{2} \\
& =\frac{1}{2 h}\left(\int_{-h}^{h} f\left(1+\alpha_{2} K\right) \alpha_{2} d \alpha_{2}-(1+K h) h q^{+}-(1-K h) h q^{-}\right) .
\end{aligned}
$$

Performing the same transformations as were used for obtaining (14), (15) we can write boundary (10) and initial (12) conditions for functions $u_{1}\left(\tau, \alpha_{1}\right), u_{2}\left(\tau, \alpha_{1}\right)$ in the form

$$
\begin{gathered}
\left.u_{1}\right|_{\alpha_{1}=\alpha_{1}^{b}}=0, \\
\left.u_{1}\right|_{\alpha_{1}=\alpha_{1}^{e}}=0, \\
\left.u_{2}\right|_{\alpha_{1}=\alpha_{1}^{b}}=0, \\
\left.u_{2}\right|_{\alpha_{1}=\alpha_{1}^{e}}=0, \\
u_{1}\left(0, \alpha_{1}\right)=\frac{1}{2 h} \int_{-h}^{h} u^{(0)} d \alpha_{2}, \\
u_{2}\left(0, \alpha_{1}\right)=\frac{3}{2 h^{2}} \int_{-h}^{h} u^{(0)}\left(1+\alpha_{2} K\right)\left(\alpha_{2}-\frac{K h^{2}}{3}\right) d \alpha_{2} .
\end{gathered}
$$

Therefore, we reduced the initial problem (9)-(12) to one-dimensional case and obtained the system of two linear differential equations for $u_{1}$ and $u_{2}(14)$, (15) with the corresponding boundary (16) and initial (17) conditions in the case of initial-boundary value problem in a thin curvilinear channel under the assumption of a linear distribution over the variable $\alpha_{2}$. 
For simplicity, let us rewrite the obtained initial-boundary value problem (14)-(17) to vector form [7]

$$
\begin{aligned}
\kappa \boldsymbol{M} \frac{\partial \boldsymbol{u}}{\partial \tau}+\frac{\kappa P e}{A} \boldsymbol{L} \frac{\partial \boldsymbol{u}}{\partial \alpha_{1}}-\frac{1}{A} & \frac{\partial}{\partial \alpha_{1}} \frac{\lambda}{A} \boldsymbol{N} \frac{\partial \boldsymbol{u}}{\partial \alpha_{1}}+\lambda \boldsymbol{P u}=\boldsymbol{f} \\
\left.\boldsymbol{u}\right|_{\alpha_{1}=\alpha_{1}^{b}} & =(0,0)^{T}, \\
\left.\boldsymbol{u}\right|_{\alpha_{1}=\alpha_{1}^{e}} & =(0,0)^{T}, \\
\left.\boldsymbol{u}\right|_{\tau=0} & =\boldsymbol{u}_{0},
\end{aligned}
$$

where $\boldsymbol{u}=\left(u_{1}, u_{2}\right)^{T}$,

$$
\begin{gathered}
\boldsymbol{M}=\left(\begin{array}{cc}
1 & \frac{K h}{3} \\
\frac{K h^{2}}{3} & \frac{h}{3}
\end{array}\right), \quad \boldsymbol{L}=\left(\begin{array}{cc}
1 & 0 \\
0 & \frac{h}{3}
\end{array}\right), \quad \boldsymbol{N}=\left(\begin{array}{cc}
1 & -\frac{K h}{3} \\
-\frac{K h^{2}}{3} & \frac{h}{3}
\end{array}\right), \quad \boldsymbol{P}=\left(\begin{array}{ll}
0 & 0 \\
0 & \frac{1}{h}
\end{array}\right), \\
\boldsymbol{f}=\frac{1}{2 h}\left(\begin{array}{c}
\int_{-h}^{h} f\left(1+\alpha_{2} K\right) d \alpha_{2}-(1+K h) q^{+}+(1-K h) q^{-} \\
\int_{-h}^{h} f\left(1+\alpha_{2} K\right) \alpha_{2} d \alpha_{2}-(1+K h) h q^{+}-(1-K h) h q^{-}
\end{array}\right),\left.\quad \boldsymbol{u}\right|_{\tau=0}=\left(\begin{array}{l}
u_{1}\left(0, \alpha_{1}\right) \\
u_{2}\left(0, \alpha_{1}\right)
\end{array}\right), \\
\boldsymbol{u}_{0}=\frac{1}{2 h}\left(\begin{array}{l}
h \\
\int_{-h}^{h} u^{(0)}\left(1+\alpha_{2} K\right)\left(\frac{3}{h} \alpha_{2}-K h\right) d \alpha_{2}
\end{array}\right) .
\end{gathered}
$$

\section{Numerical experiments}

For comparison we solve the problem (18)-(20) using MsFEM and standard FEM methods. We present the multiscale basis functions in vector form, so they can be found from (3)

$$
\boldsymbol{\varphi}_{i}=\left(\varphi_{i}^{1}, \varphi_{i}^{2}\right)^{T}, \quad i=\overline{1, n},
$$

where $n$ - count of finite elements on coarse grid.
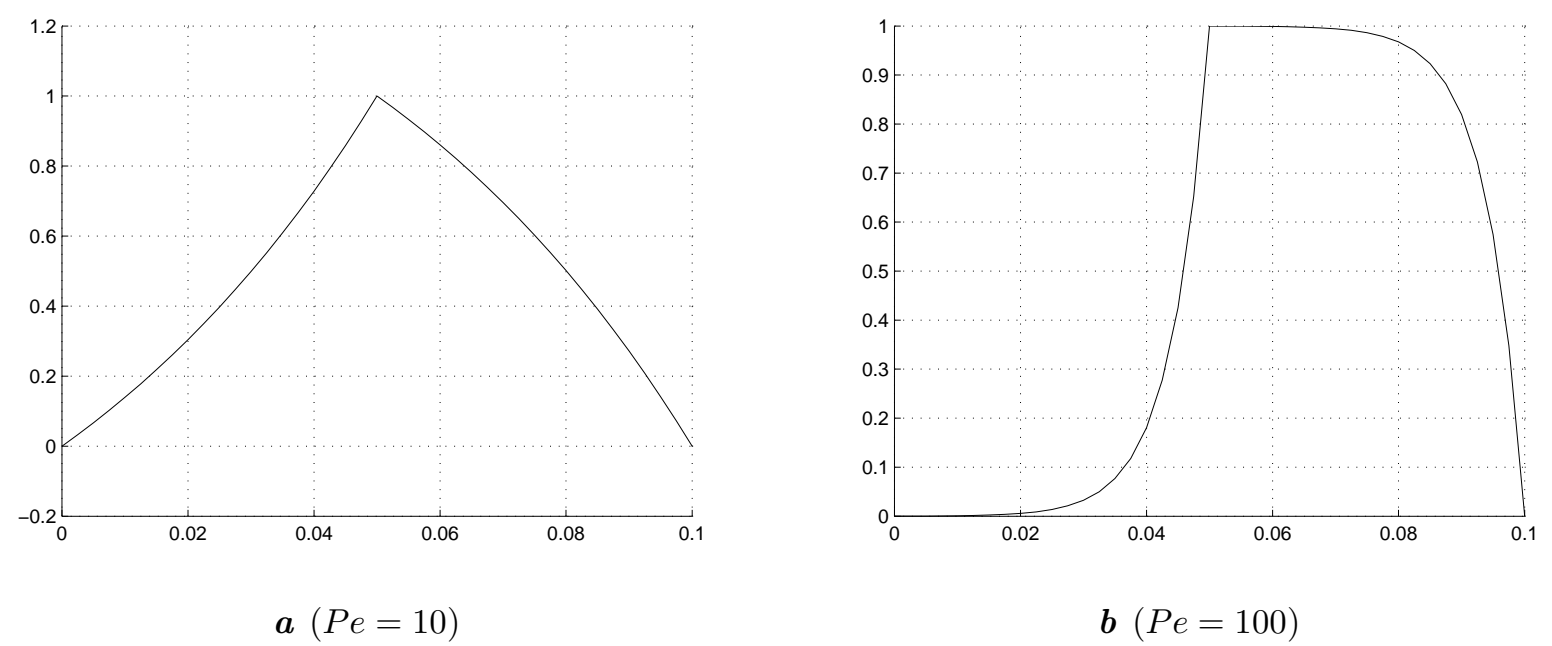

Fig. 2. Multiscale basis functions obtained in rectilinear channel with 20 finite elements for coarse and fine grids. 
In Fig. $2 a$ multiscale basis functions for (14) are presented for $P e=10$ and $P e=100$ respectively. Note that for low Peclet numbers multiscale basis functions are similar to piecewise linear basis functions of FEM, so we expect that the solution obtained by MsFEM will behave in the same manner as the FEM solution for low Peclet numbers. More interesting is the case of sufficiently large Peclet numbers, where multiscale basis functions are completely different from the FEM basis.

We use the following values of parameters for numerical experiments: $\alpha_{1}^{b}=0, \alpha_{1}^{e}=1, h=0.15$, $\kappa=1, \lambda=0.6, f=1, q^{+}=q^{-}=-1$. At first we consider the rectilinear channel, that is when $A=1$ and $K=0$. In this case the analytical solution can be obtained for the stationary problem, so we can compare MsFEM and FEM solutions with the analytical one and make conclusions about efficiency of these methods.

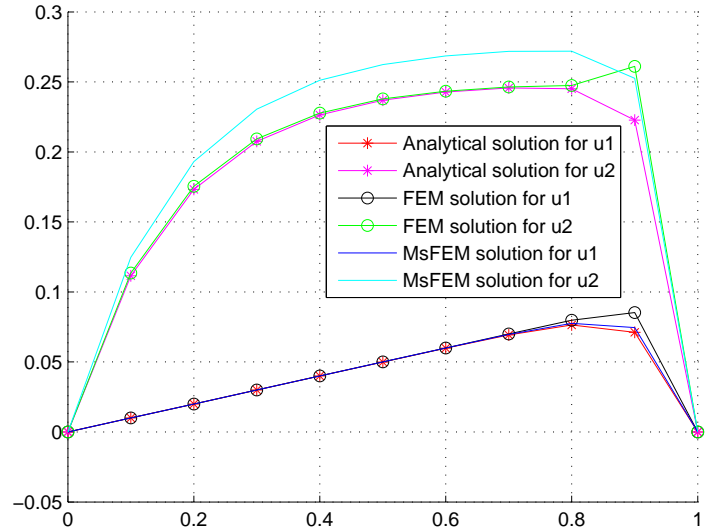

$\boldsymbol{a}(n=10)$

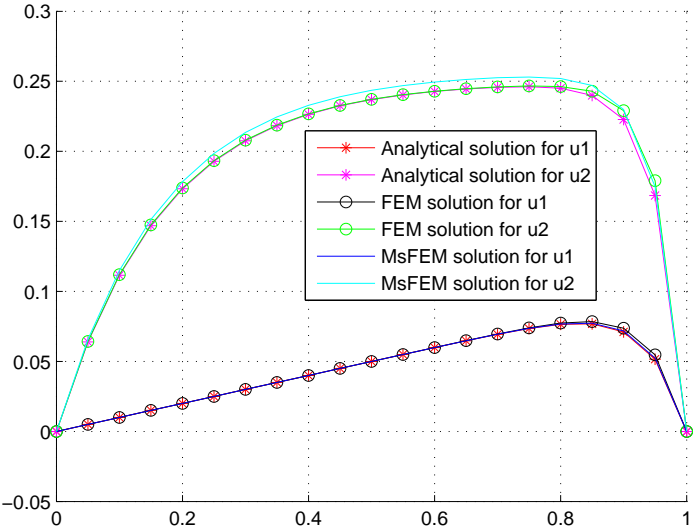

$\boldsymbol{b}(n=20)$

Fig. 3. Solutions obtained in rectilinear channel with $P e=10$ and $n$ finite elements for coarse and fine grids.

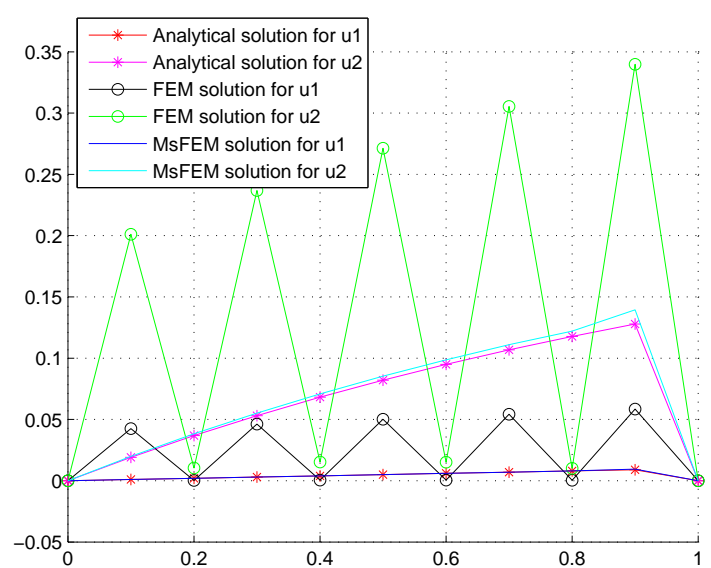

$\boldsymbol{a}(n=10)$

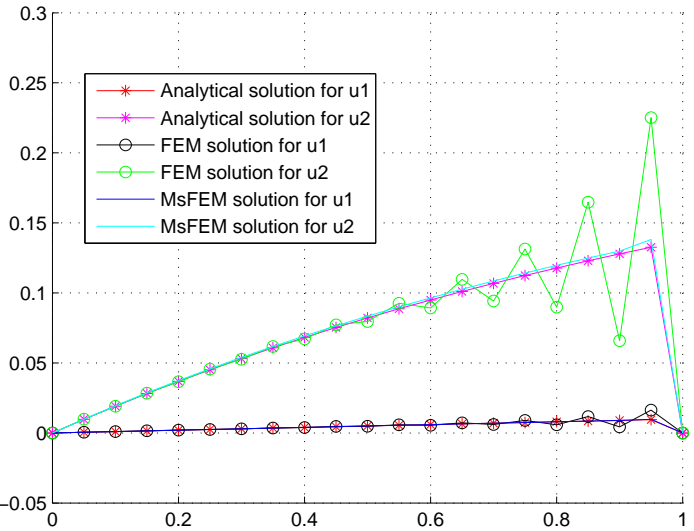

$\boldsymbol{b}(n=20)$

Fig. 4. Solutions obtained in rectilinear channel with $P e=100$ and $n$ finite elements for coarse and fine grids.

As we can see from Figs. 3-4 the results obtained by MsFEM and FEM methods for $P e=10$ are almost the same, while for $P e=100$ they are completely different (as was expected from the form of constructed multiscale basis functions). For sufficiently large Peclet numbers the MsFEM solution is much more accurate and stable, while the solution obtained by FEM contains unnatural oscillations. 
The absolute errors for FEM and MsFEM solutions (see Table 1) confirm that MsFEM is efficient even for small amount of finite elements.

Table 1. Absolute errors of FEM and MsFEM solutions for $P e=100, n=10$.

\begin{tabular}{|c|c|c|c|c|}
\hline Node & $u_{1}$ FEM & $u_{1}$ MsFEM, 1.0e-0 ${ }^{*}$ & $u_{2}$ FEM & $u_{2}$ MsFEM \\
\hline 0.1 & 0.0415 & 0.0000 & 0.1820 & 0.0008 \\
0.2 & 0.0017 & 0.0000 & 0.0265 & 0.0015 \\
0.3 & 0.0433 & 0.0000 & 0.1837 & 0.0021 \\
0.4 & 0.0035 & 0.0000 & 0.0529 & 0.0027 \\
0.5 & 0.0452 & 0.0000 & 0.1892 & 0.0032 \\
0.6 & 0.0055 & 0.0000 & 0.0798 & 0.0037 \\
0.7 & 0.0472 & 0.0010 & 0.1985 & 0.0042 \\
0.8 & 0.0077 & 0.0222 & 0.1077 & 0.0043 \\
0.9 & 0.0495 & 0.4712 & 0.2118 & 0.0116 \\
\hline \hline Max & 0.0495 & 0.4712 & 0.2118 & 0.0116 \\
\hline
\end{tabular}

Next we consider a simple parabolic channel, the middle curve of which can be described by equation $y=x^{2}$. In this case $A=\sqrt{1+4 x^{2}}$ and $K=\frac{2}{\left(\sqrt{1+4 x^{2}}\right)^{3}}$. The results obtained for this case are presented in Figs. 5-6.
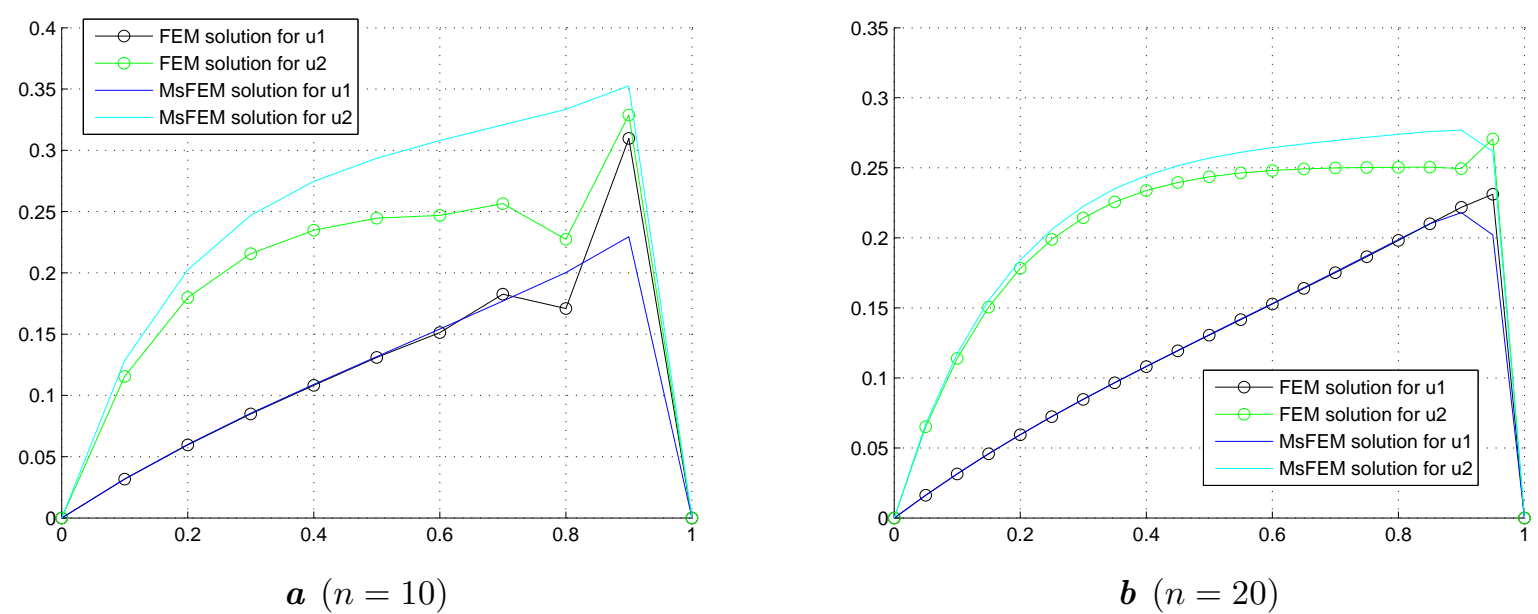

Fig. 5. Solutions obtained in parabolic channel with $P e=10$ and $n$ finite elements for coarse and fine grids.
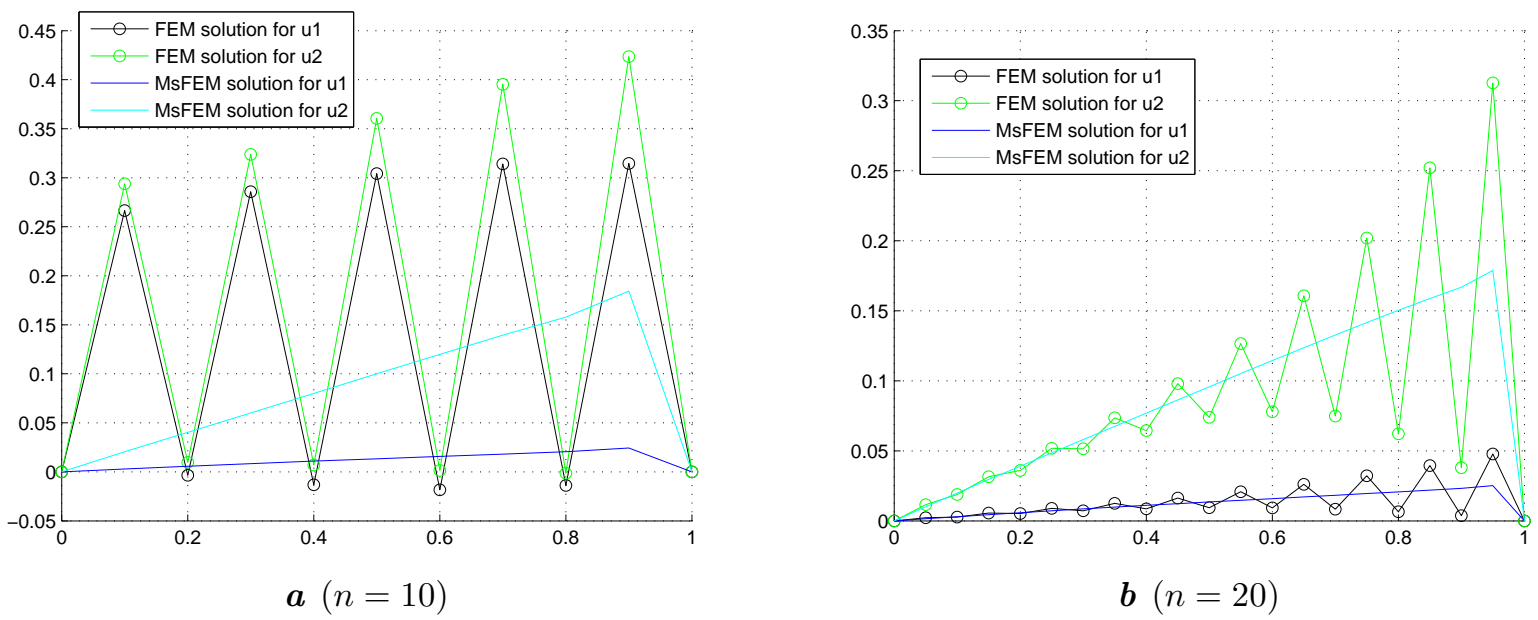

Fig. 6. Solutions obtained in parabolic channel with $P e=100$ and $n$ finite elements for coarse and fine grids. 
Now we can see that in the case of parabolic channel both solutions obtained by MsFEM and FEM converge and become more stable while increasing the amount of finite elements. But in the case when Peclet number is large the MsFEM solution is stable even for small amount of finite elements, while the solution obtained by FEM is oscillating and unnatural. The obtained results demonstrate the efficiency of MsFEM for large Peclet numbers.

The Crank-Nicholson scheme is used for solving the non-stationary problem. In Fig. 7 we can see MsFEM solutions in different points of time. Here $P e=100, n=10$ and step of time discretization is 0.01 . Fig. 7 shows that the presented solutions converge.

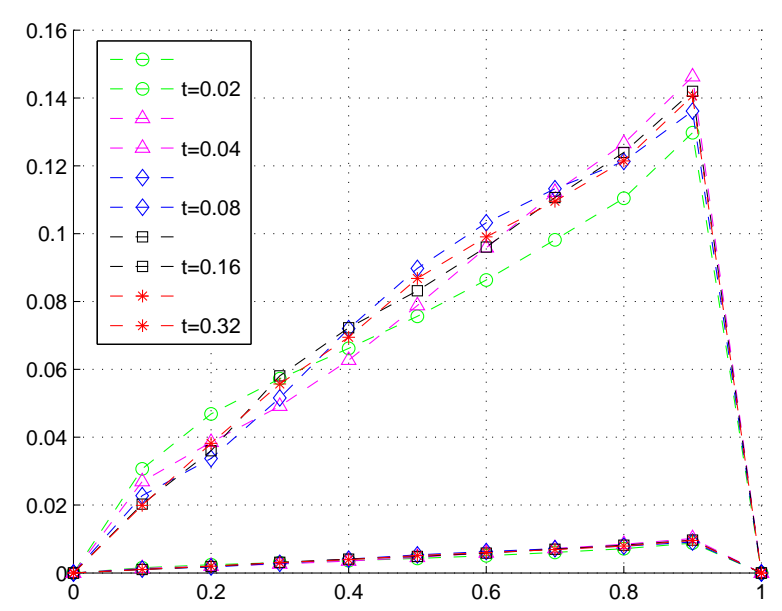

Fig. 7. MsFEM solutions in different time moments.

\section{Conclusions}

The multiscale finite element method was applied to solving the advection-diffusion problem with large Peclet numbers in a thin curvilinear channel. The solutions were also obtained by FEM and analytically (in the case of stationary advection-diffusion problem) for comparison and verification of the results. It was shown that the MsFEM solution is stable and matches the analytical solution in the case of large Peclet numbers, while the FEM solution contains unnatural oscillations. Hence MsFEM allows to solve the advection-diffusion problem with large Peclet numbers more accurately even with a small number of finite elements.

[1] Savula Ya. Numerical analysis of problems of mathematical physics by variational methods. Lviv, $221 \mathrm{p}$. (2004).

[2] Efendiev Y., Hou T. Multiscale finite element methods. Theory and application. NY, Springer (Surveys and Tutorials in the Applied Mathematical Sciences). Vol.4, 234 p. (2009).

[3] Spodar N., Savula Ya. Application of multiscale finite element method for solving the one-dimensional advection-diffusion problem. Physico-mathematical modelling and informational technologies. 19, 190-197 (2014).

[4] Spodar N., Savula Ya. Computational aspects of multiscale finite element method. Physico-mathematical modelling and informational technologies. 23, 169-177 (2016).

[5] Rashevskij P. Course of differential geometry. Moscow, Leningrad (State publishing house of technical and theoretical literature), 3th edition, recycled, 428 p. (1950).

[6] Savula Ya. H., Koukharskyi V. M., Chaplia Ye. Ya. Numerical analysis of advection-diffusion in the continuum with thin canal. Numerical Heat Transfer, Part A: Applications: An International Journal of Computation and Methodology. 33 (3), 341-351 (1998).

[7] Kukharskyy V., Kukharska N., Savula Ya. Application of Heterogeneous Mathematical Models for the Solving of Heat and Mass Transfer Problems in Environments with Thin Heterogeneties. Physico-mathematical modelling and informational technologies. 4, 132-141 (2006). 


\title{
Числовий аналіз задач адвекції-дифузії у тонкому криволінійному каналі на основі різномасштабного методу скінченних елементів
}

\author{
Мазуряк Н., Савула Я. \\ Лъвівсъкий національний університет імені Івана Франка \\ вул. Університетсъка, 1, 79000, Лъвів, Україна
}

Розглянуто задачу адвекції-дифузії у тонкому криволінійному каналі. До розв'язування цієї задачі застовано різномасштабний метод скінченних елементів. Показано, що цей метод є ефективним для достатньо великих чисел Пекле. Наведено та проаналізовано результати обчислювальних експериментів.

Ключові слова: різномасштабний метод скінченних елементів, адвекиіл-дифузіл, тонкий криволінійний канал.

2000 MSC: $65 \mathrm{M} 55,65 \mathrm{M} 60$

УдК: $517.958: 519.6$

Mathematical Modeling and Computing, Vol. 4, No. 1, pp. 59-68 (2017) 